\title{
Acontecimentos que fizeram a história da oncologia no Brasil: Instituto Nacional de Câncer (INCA)
}

Occurrences that made the history of oncology in Brazil: National Cancer Institute (INCA)

\author{
Eliana Maria Teixeira Barreto
}

\section{Resumo}

O conhecimento acerca dos acontecimentos que antecederam a criação e o funcionamento do Instituto Nacional de Câncer (INCA) torna-se importante para que se possa compreendê-los sob a perspectiva histórica, considerando que tiveram início na década de 20. Neste período, surgiram as primeiras políticas públicas voltadas para o tratamento e controle do câncer, e medidas governamentais que ensejaram e/ou apoiaram outras iniciativas em prol da luta contra a doença no país, tornando relevante a atuação do INCA no campo da oncologia. Este artigo trata dessa reconstrução histórica.

Palavras-chave: Oncologia; História; Institutos de câncer; Política de saúde; Brasil.

\section{Abstract}

The knowledge of the facts that preceded the creation of the National Cancer Institute (INCA) is important to understand the historical perspective in which it occurred, in the 20 's. In this period appeared the first public policies aiming cancer treatment and control, as well as the actions fighting the disease. This paper reconstructs this history showing the relevance of the INCA in this scenario.

Key words: Oncology; History; Cancer care facilities; Health policy; Brazil.

Enfermeira do Centro de Transplante de Medula Óssea, INCA; Mestre em Enfermagem- Escola de Enfermagem Anna Nery da Universidade Federal do Rio de Janeiro.

Endereço para correspondência: Eliana M. T. Barreto, CEMO, Pça da Cruz Vermelha, 23, 7 andar. 20230-130, Centro, Rio de Janeiro, RJ 


\section{INTRODUÇÃO}

As primeiras iniciativas para o controle do câncer no Brasil remontam ao início do século 20, orientadas quase que exclusivamente para o diagnóstico e tratamento. Pouca ênfase era dada à prevenção, pela escassez de conhecimento sobre a etiologia da doença. ${ }^{1} \mathrm{Na}$ opinião dos especialistas em oncologia, deveria haver uma preocupação maior com a prevenção e o diagnóstico precoce, com o que concordavam os cirurgiôes.

$\mathrm{Na}$ ocasião, o câncer e muitas outras doenças assolavam a cidade do Rio de Janeiro. No sentido de evitar que se propagassem, o governo assumiu a assistência à saúde através de diversas medidas, com destaque para a criação de serviços públicos, a vigilância sanitária e o controle mais eficaz sobre os portos.

Em 1920 foi criado o Departamento Nacional de Saúde Pública (DNSP), visando a ampliação do campo de ação assistencial, abrangendo a saúde infantil, industrial e ocupacional. Com isso, coube à União promover e regular os serviços de saúde no território nacional. Em 1923, este Órgão passou a chamar-se Departamento Nacional de Saúde (DNS).

De acordo com relatos de Mário Kroeff ${ }^{2}$, "[...] data de 1921 o primeiro movimento em São Paulo, de alcance social, no tocante ao problema do câncer [...]". O prestigiado cancerologista assinalou que "[...] por deliberação do Dr. Artur Bernardes, e segundo os planos do Professor Borges da Costa [...]", foi instalado naquele ano, em Belo Horizonte, o Instituto de Radium, o pioneiro do país destinado aos portadores de neoplasias.

Mas, para atender às reivindicações da classe operária no que se referia à saúde, o Estado implantou um regime que, subseqüentemente, resultou na criação de uma previdência / assistência social nacional, iniciando um sistema baseado nas experiências e nos resultados de serviços vigentes em outros países capitalistas, originando as Caixas de Aposentadoria e Pensōes (CAPs).

A primeira categoria profissional contemplada pelo novo sistema foi a dos ferroviários. Posteriormente, outras foram favorecidas, como as dos portuários, dos marítimos, dos servidores públicos, dos operários de mineradoras e dos transportadores de cargas. Este projeto foi regulamentado em 1923, pelo Decreto no 4.682, que ficou conhecido como "Lei Eloy Chaves". 3

Em 1924, durante o II Congresso Brasileiro de Higiene, comparou-se o declínio da tuberculose com o aumento do câncer nos principais centros urbanos do Brasil.1 O índice de mortalidade pela doença no país era considerado baixo, mas havia a tendência de elevar-se a cada década, caso não fossem adotadas providências a respeito. $\mathrm{O}$ assunto despertou a atenção do Dr. Carlos
Chagas, diretor da Divisão Nacional de Saúde, que codificou o interesse da comunidade médica voltada para o tema através de regulamentação sanitária, fixando cláusulas para a luta contra o câncer.

Deve-se ressaltar, porém, que a Reforma Carlos Chagas, iniciada em 1921, já previa a elaboração de estatísticas sobre o câncer, com a recomendação de que os atestados de óbito fossem lavrados em impressos apropriados contendo quesitos sobre a doença, medida que consolidou o incentivo à notificação dos casos de câncer. Todavia, somente em 1983 foi implantado o Primeiro Registro Hospitalar de Câncer do Brasil, no Instituto Nacional de Câncer (INCA), de acordo com as normas padronizadas e preconizadas pela Organização Pan Americana de Saúde (OPAS), com apoio da Agência Internacional de Pesquisa em Câncer (IARC). ${ }^{1}$

$\mathrm{Na}$ década de 30, as medidas adotadas pelo Estado em relação às atividades de saúde sob a ingerência do Ministério da Justiça e Negócios Interiores, passaram à responsabilidade do recém-criado Ministério da Educação e Saúde, cujas ações promoveriam, a partir de então, mudanças significativas na área de saúde através da organização, em âmbito estadual, dos departamentos de saúde e da criação de hospitais, centros e postos de saúde sob a coordenação do DNS.

No final desta década criou-se o Conselho Nacional de Saúde (CNS) com a finalidade de prestar assessoria ao Ministério da Educação e Saúde. Um dos atos mais importantes do CNS foi sugerir a incorporação das estruturas das CAPs aos Institutos de Aposentadoria e Pensōes (IAPs). ${ }^{3}$

Durante o governo de Getúlio Vargas (1930-1945), a sociedade civil reivindicou algumas açôes de combate ao câncer e de atendimento às necessidades da população. Levando em consideração esses anseios, o Governo adotou uma política nacional voltada para o controle da doença. Como consequiência, foi criado, em 1937, o Centro de Cancerologia do Serviço de Assistência Hospitalar do Distrito Federal (Rio de Janeiro), que veio a se constituir no atual Instituto Nacional de Câncer (INCA). Segundo relato de um de seus fundadores, a criação do Órgão prestigiou os serviços médicos dispensados aos cancerosos, a formação de recursos humanos para o setor e também a área de pesquisa básica. ${ }^{2}$

O evento concretizou o desejo do Dr. Mário Kroeff, renomado médico cancerologista que muito trabalhou em prol desta causa, e que sempre opinara no sentido de que "deveria haver um órgão que, administrativamente, seria chamado Instituto Nacional de Câncer, órgão técnico encarregado do diagnóstico e tratamento dos doentes, e que instruísse outros médicos, como acontece até hoje". ${ }^{4}$

$\mathrm{Na}$ década de 40, as iniciativas governamentais 
voltaram-se para a reformulação e ampliação das diversas organizações de saúde. Assim, toda atenção nessa área passou a ser concentrada no Ministério da Educação e Saúde, principalmente aquelas relacionadas ao combate às endemias rurais e proteção à maternidade e à infância.

A necessidade de ampliar as açôes na luta contra o câncer levou à criação, em 1941, do Serviço Nacional de Câncer (SNC), cujos objetivos eram organizar, orientar e controlar a doença no País. Foi relevante, também, a criação, em 1942, do Serviço Especial de Saúde Pública (SESP), cujas funçôes eram atender às questôes de saúde em áreas estratégias militares e econômica, como as de produção de borracha no Vale do Amazonas, e de minérios no Vale do Rio Doce. Posteriormente, com recursos financeiros oriundos da Fundação Rockefeller (EUA), o SESP ampliou e expandiu seus serviços nas regiōes Nordeste e Norte do país. ${ }^{3}$

De 1942 a 1946, diversos movimentos sociais originaram a Liga Bahiana Contra o Câncer e a Associação Paulista de Combate ao Câncer, que organizaram e desenvolveram atividades de grande relevância para a assistência aos portadores de neoplasias. Em 1947, a Liga Paranaense Contra o Câncer teve papel de destaque nesse processo e, junto a outras campanhas, também obteve recursos financeiros. Posteriormente, a Associação Paulista de Combate ao Câncer investiu considerável montante na construção, em São Paulo, do Instituto Central, inaugurado em 1953, atual Hospital "Antonio Cândido Camargo".5

As iniciativas filantrópicas e governamentais possibilitaram a organização das ligas contra o câncer e o envio de médicos sanitaristas e cancerologistas ao exterior para ampliarem seus conhecimentos científicos, confirmando o interesse que o assunto suscitava na sociedade. Essas políticas de saúde tiverem o apoio das Ligas Contra o Câncer e de Fundaçôes, como a Martinelli e a Laureano, que se destacaram na campanha contra a doença através de conferências, exposiçōes e arrecadação de fundos. ${ }^{2}$

O reflexo dos avanços tecnológicos e científicos existentes nos países desenvolvidos resultou em ações que levaram ao aprimoramento dos modelos de tratamento já incorporados pela comunidade médica sanitarista e cancerologista brasileira, cujos saberes também foram influenciados pelas experiências vivenciadas na realidade norte-americana.

Registra-se, ainda em 1947, a criação do Plano SALTE (Saúde, Alimentação, Transporte e Energia), cuja meta era a redistribuição dos médicos pelo país, constituindose no primeiro plano integral relacionado ao desenvolvimento sócio-econômico no Brasil. Embora este projeto tenha recebido apoio orçamentário federal, não obteve o sucesso esperado, nem chegou a ser totalmente implantado. ${ }^{6}$

Na década de 50, a atenção governamental enfocou a modernização dos cuidados médico-sanitários e o uso de medicamentos e equipamentos hospitalares em toda área de saúde. Como conseqüência, houve o crescimento da indústria químico-farmacêutica e modificações de grande relevância na política de saúde, em especial as do câncer, em conseqüência da complexidade do seu diagnóstico e tratamento.

Deve-se ressaltar a criação do Ministério da Saúde (MS), em 1953, pelo Decreto-Lei no 1.920 , desmembrado do Ministério da Educação e Saúde, que manteve a estrutura básica do Departamento Nacional de Saúde, conservando o Serviço Nacional de Câncer.7 No mesmo ano, completou-se o processo de fusão das CAPs, que deu origem a uma entidade única, depois transformada no Instituto de Aposentadoria e Pensões dos Ferroviários e Empregados em Transportes e Cargas. ${ }^{3}$

Em 1956 foi criada uma Unidade da Fundação das Pioneiras Sociais, objetivando o atendimento ambulatorial. Vale esclarecer que, posteriormente, esta Unidade especializou-se em câncer ginecológico e mamário, cuja necessidade de expansão do atendimento ambulatorial ensejou a criação, em 1977, de uma Unidade Hospitalar de Internação denominada Instituto Nacional de Ginecologia Preventiva e de Reprodução Humana. Sua extinção deu lugar ao surgimento do Hospital Luiza Gomes de Lemos, com atividades ambulatoriais e hospitalares voltadas para o tratamento daquelas patologias.

Nos anos 60 surgiram alguns movimentos políticos que apontavam para o início de um processo objetivando estabelecer o conceito público de bem-estar social no País, mas o golpe militar de 1964, com a edição do Ato Institucional no 5 , no mesmo ano, ${ }^{6}$ interrompeu este projeto, culminando por favorecer a instalação e o crescimento das empresas privadas na área da saúde, sem o devido controle por parte das autoridades governamentais militares no poder, fato que contribuiu para a ocorrência de irregularidades na rede privada, cujos hospitais e clínicas recebiam financiamento da Previdência Social e mantinham ênfase na medicina curativa.

Nessa ocasião, em virtude da redução das verbas destinadas ao MS, o setor previdenciário sofreu acentuada decadência, atingindo diretamente a área da Saúde Pública, prejudicando os serviços de controle das doenças endêmicas, alguns dos quais foram desativados ou continuaram a funcionar precariamente. A Saúde Pública viu-se abalada por irregularidades constantes, pela falta de investimentos e gerenciamento inadequado das verbas disponíveis. 
Em 21 de novembro de 1966, como resultado da fusão dos Institutos de Aposentadorias e Pensōes, foi criado o Instituto Nacional de Previdência Social (INPS) através do Decreto-Lei no 72 , que proporcionou a reorganização e reformulação do modelo assistencial vigente. ${ }^{3}$

No bojo dos acontecimentos, e visando dinamizar as atividades de cunho oncológico, o Serviço Nacional de Câncer decidiu institucionalizar a Campanha Nacional de Combate ao Câncer (CNCC), o que se efetivou em 1967. A CNCC teve como objetivos básicos intensificar e coordenar, no território brasileiro, as atividades desenvolvidas por instituições públicas e privadas, atuando nas áreas de prevenção, diagnóstico, assistência médica, formação de técnicos especializados, ação social, reabilitação e pesquisas relacionadas com a neoplasia. Procurava-se, assim, reduzir a incidência e o índice de mortalidade por câncer. Todavia, apesar da amplitude e importância do programa, e da ênfase dada à questão, os resultados desta batalha foram significativamente reduzidos pela falta de recursos financeiros.

Em maio de 1969, por força de ato ministerial, o INCA foi desligado do Ministério da Saúde, passando para a administração da Fundação Escola de Medicina e Cirurgia do Rio de Janeiro, ligada ao Ministério da Educação e Cultura (MEC). ${ }^{8}$ Todavia, movimentos internos e externos ocorreram visando a sua reincorporação àquele Ministério, o que só ocorreu com a promulgação da Lei no 5.734 , de 16 de novembro de 1971, com vigência a partir de $1^{\circ}$ de janeiro de 1972.

Em janeiro de 1972, com a edição da Portaria GB-30 de 19 daquele mês, o INCA passou a subordinar-se ao Gabinete do Ministro da Saúde. Em maio do mesmo ano, o Órgão governamental divulgou esclarecimentos à população acerca da atuação da CNCC no Rio de Janeiro, cujo objetivo era melhorar o nível de assistência à população. Essa visão otimista centrava-se nos recursos advindos da CNCC destinados a reorganizar a infraestrutura do INCA e reequipar o Serviço, possibilitando o acompanhamento dos avanços tecnológicos da época. Em 22 de setembro, pela Portaria 18-BR do Ministério da Saúde, o Instituto Nacional de Câncer foi reintegrado à Divisão Nacional de Saúde. ${ }^{5}$

Em 1973, o INCA elaborou um sistema de estudos baseados em pesquisas já realizadas em instituições internacionais. Estas pesquisas visavam a implantação e implementação do uso de drogas anti-cancerígenas, em fase de estudos experimentais no país, embora na América do Norte o uso das mesmas demonstrasse resultados favoráveis pautados na utilização do "Separador de Células e seu Uso no Tratamento de Apoio de Isolamento no Ambiente Livre de Germes", uma tecnologia avançada para aquela época no Brasil.
Em 1974 foram criados o Ministério da Previdência e Assistência Social (MPAS), voltado para o seguro social e a assistência médica, o Conselho de Desenvolvimento Social e o Fundo de Apoio ao Desenvolvimento Social (FAS). Pode-se perceber que nos órgãos governamentais decisórios, era clara a opção pelo modelo privado de assistência, "baseado na medicina terapêutica, voltada para o indivíduo, utilizando alta tecnologia e requerendo recursos humanos especializados". 5

Neste mesmo ano, os médicos do INCA passaram a protocolar e a classificar as neoplasias de acordo com as características já identificadas, com base em estudos realizados no próprio Instituto.

Entre 1974 e 1978, o PNCC foi absorvido pelo II Plano Nacional de Desenvolvimento, que dispunha de verba específica para sua implantação e desenvolvimento. Como instrumento administrativo e gestor deste Plano, foi designada a Campanha Nacional de Combate ao Câncer (CNCC). Por seu intermédio, foram adquiridos equipamentos como bombas de cobalto, agulhas de césio e outros, para vários hospitais das redes pública e privada, beneficiados por meio de cessão do material em regime de comodato. A esse respeito, assim se pronunciou o Dr. Adib Domingos Jatene:

[...] Não é absurdo dizer que quem orienta a medicina, hoje - a chamada medicina moderna -, não são mais os médicos, nem as organizações hospitalares. Quem orienta a medicina é a indústria. Porque a indústria, atuando como tal, com as suas técnicas de marketing [...] induz os hospitais, as faculdades, os profissionais de saúde, os professores e os alunos à utilização de um arsenal que, realmente, colocado a serviço do homem, melhora suas perspectivas de vida. [...] $]^{7}$

A opinião do prestigiado médico estava correta porque a União já investira cerca de 430 milhōes, sem contar com os 10 milhôes de dólares decorrentes de empréstimo externo aplicados principalmente em equipamentos em comodato nas instituições de câncer, o que levou a novas discussóes a respeito das linhas de ação da DNDCD, que passava a ser vista apenas como "uma divisão do Ministério da Saúde com papel normativo e de orientação, sem recursos orçamentários próprios". 5

$\mathrm{Na}$ opinião de Edmur Flávio Pastorello, à época diretor do DNDCD,

[...] o câncer era um problema de saúde pública [...] um problema fundamentalmente hospitalar. Nessa medida, haveria que se recuperar o INCA, o que passava por uma redefinição da rede hospitalar do Ministério da Saúde $[\ldots] .^{5}$ 
Em 1975, o convênio firmado entre o Ministério da Previdência e Assistência Social (MPAS) e o MS teve como fruto a proposta, no âmbito da Previdência, da criação de um Programa de Controle ao Câncer (PCC), universalizando os procedimentos relacionados à doença e instituindo Comissões de Oncologia em nível local, regional e nacional, com vistas a integrar as ações de controle do câncer. ${ }^{4}$ No desenvolvimento deste Programa, a DNC foi transformada em Divisão Nacional de Doenças Crônico-Degenerativas (DNDCD), o que possibilitou a ampliação das açóes da extinta DNC.

As políticas de saúde caminharam em paralelo durante este processo evolutivo, no interesse de oferecer melhor qualidade de vida à população, especialmente para a classe menos favorecida social e economicamente.

Deve-se enfatizar que o discurso oficial do governo sofreu considerável influência das idéias dos oposicionistas, solidificadas em propostas modernas como as que foram apresentadas na Conferência Internacional de Alma-Ata (1978), que direcionou toda atenção aos cuidados primários em saúde, tendo sido este ponto considerado estratégico para alcançar saúde melhor para todos. Esta Conferência ocorreu na União das Repúblicas Socialistas Soviéticas (URSS), onde a Organização Mundial de Saúde (OMS) aprovou e consagrou uma proposta acerca da construção de novas políticas de saúde, enviada a todos os governos do mundo, considerando os cuidados primários como ponto básico para atingir a meta social de "Saúde para todos no ano 2000", em especial nos países subdesenvolvidos. ${ }^{3}$

Em 1977, a Lei no 6.439 instituiu o Sistema Nacional de Previdência e Assistência Social (SINPAS), em cuja estrutura incluía-se o Instituto Nacional de Assistência Médica da Previdência Social (INAMPS). ${ }^{3}$

Em 1978 foi realizada em Brasília (Distrito Federal) a VII Conferência Nacional de Saúde. O evento promoveu e debateu temas amplos relacionados à implantação e ao desenvolvimento do Programa de Serviços Básicos de Saúde (PREVSAÚDE), sob a égide dos Ministérios da Saúde e da Previdência e Assistência Social,7 destacando-se as condições sanitárias e de habitação, alimentação e nutrição da população, além do incentivo aos programas de vigilância epidemiológica.

A década de 70 teve como característica marcante o "milagre econômico", que entusiasmou a classe média. Prevalecia a política de fornecer à sociedade matériaprima e produtos semi-elaborados, com destaque para a importância de máquinas industriais. Mas esse processo não trouxe os benefícios almejados pela população brasileira em relação ao seu desenvolvimento econômico. Além do mais, no que tange aos serviços hospitalares, era grande ainda o contingente de desassistidos em decorrência da escassez de recursos oferecidos pelos serviços de Saúde Pública, configurando um quadro nosológico que abrangia doenças típicas que acometiam ricos e pobres.

$\mathrm{Na}$ década de 80, altas taxas de desemprego, medidas recessivas e perda do poder aquisitivo do salário mínimo foram fatores determinantes de grande retrocesso sóciopolítico, que resultaram no decréscimo do desenvolvimento econômico e no ressurgimento da inflação. O período ficou conhecido como "A Década Perdida", devido ao aumento da pobreza e da miséria da população brasileira, sobretudo daquela menos favorecida. ${ }^{9}$

O agravamento desta crise financeira atingiu o INAMPS, que precisou equacioná-la como gestor da assistência médica aos segurados da Previdência e como responsável pela assistência médica individual à população. A situação foi enfrentada num contexto não apenas da extensão dos benefícios a alguns setores, mas abarcando a universalização do direito à saúde e do acesso aos serviços. Emergiram do sistema gestor de saúde diversos projetos de expansão dos serviços com a intenção de atender toda a população, tendo como enfoque a Saúde Pública.

Os planos governamentais elaborados visavam a integração das assistências preventiva e curativa. A estratégia adotada possibilitou, de um lado, o controle dos gastos pelo combate às fraudes; de outro, a contenção da expansão dos contratos assistenciais de saúde com prestadores privados, privilegiando o setor público. $\mathrm{O}$ INAMPS iniciou um processo de integração da rede hospitalar que culminou por eliminar a diferença entre as clientelas segurada e não segurada, para fins de atendimento de saúde. Tornou-se necessário reorientar a política global para o setor, articular os dois Ministérios (Saúde e Previdência) e promover a coordenação dos mesmos com os níveis estaduais e locais.

A criação da Comissão Interministerial de Planejamento (CIPLAN) deu-se em 1980, com a principal função de fixar os repasses federais daqueles Ministérios para financiamento de serviços prestados pelas redes estaduais e municipais, além de estabelecer normas de articulação programática entre os Ministério da Saúde, da Previdência e da Educação (Hospitais Universitários e de Ensino). A CIPLAN era presidida pelos secretários-gerais dos Ministérios da Saúde e da Previdência Social, e composta por representantes desses Ministérios e do Ministério da Educação. ${ }^{4}$

$\mathrm{O}$ regime de co-gestão consistia, basicamente, na 
participação igualitária do INAMPS e do Ministério da Saúde do custeio do programa, criando-se paralelamente, um Conselho Técnico-Administrativo (CTA), com representantes das entidades envolvidas e financiadores. ${ }^{7}$ Este regime teve bons resultados e oportunizou à população realizar maior número de exames e de aplicaçōes radioterápicas, isto porque houve "melhor aproveitamento dos recursos disponíveis", 5 o que permitiu ao INCa desenvolver o Programa Integrado de Medicina Nuclear, Radioterapia e Atividades Afins (PIMN), em colaboração com o Hospital dos Servidores do Estado (HSE) e interveniência da CNCC.

A acentuada situação de crise financeira na Previdência Social acabou por conduzir a uma nova tentativa de racionalizar a assistência no setor saúde, levada a efeito pela criação, em setembro de 1981, do Conselho Consultivo da Administração de Saúde Previdenciária (CONASP), através do Decreto no 86.329, priorizando as ações primárias, com enfoque na assistência ambulatorial e na integração das instituiçōes federais, estaduais e municipais com vistas a um sistema regionalizado e hierarquizado de saúde, constituindo-se nas Ações Integradas de Saúde (AIS).

A implantação das AIS foi um dos projetos elaborados pelo CONASP, representando o primeiro grande movimento de mudanças gerenciais e de alcance nacional, com repercussōes sólidas do Sistema Nacional de Saúde do país. Este projeto, amplamente divulgado em 1984, trouxe novas perspectivas para o setor saúde, e ofereceu possibilidades de mudanças, implantadas posteriormente em todos os Estados e em diversos municípios. Com essa estratégia, o trabalhador da área rural passou a ter acesso aos postos de saúde e aos hospitais do INAMPS o que, até então, não lhe era possível. Em âmbito estadual, foram instaladas as Comissões Interinstitucionais de Saúde (CIS). Com isso, o volume de serviços assistenciais prestados cresceu no decorrer de dois anos. Seguiram-se reformas estruturais e a realização de programas e convênios técnicocientíficos que culminaram por projetar o INCA como centro especializado de ensino e pesquisa oncológica. $\mathrm{Na}$ ocasião, foram contratados profissionais administrativos e técnicos para dar conta dos diferentes projetos e/ou serviços que estavam em andamento ou em plena fase de implantação.

Um desses projetos referia-se à criação e implantação do Centro Nacional de Transplante de Medula Óssea (CEMO), visto como um desafio institucional porque seriam necessárias obras para adequar o espaço físico do INCA, o que foi levado a efeito pelo Dr. Ary Frauzino Pereira, então diretor da Instituição, com auxílio de verba oriunda do Fundo de Apoio ao Desenvolvimento
Social (FAS). Um dos motivos da criação do CEMO era permitir que pacientes oncológicos tivessem acesso, no Brasil, ao tratamento adequado ao seu problema de saúde que, na época, só era passível de realização no exterior, mediante encaminhamento pela Previdência, ao custo de mais de vinte milhões de cruzeiros cada um. A Resolução CIPLAN no 12/82 autorizou a criação do CEMO (inaugurado em junho de 1983), o que foi considerado um marco na área da oncologia.

Reconhecendo, porém, que ações de diagnóstico e tratamento (controle), dissociadas das ações de prevenção e diagnóstico precoce, não alterariam o perfil da mortalidade por câncer no País, em 1986 a CNCC criou o Sistema Integrado e Regionalizado de Controle do Câncer (SIRCC) como estratégia para viabilizar as ações de controle da doença no Rio de Janeiro.

Em 1987, o Programa de Oncologia (PRO-ONCO) da CNCC foi institucionalizado com o objetivo de implantar as açōes previstas no SIRCC, em âmbito nacional. Em 1990, já com a Nova Lei Orgânica da Saúde e a Reforma Sanitária em vigor, o Ministério da Saúde sofreu nova reformulação,

$$
\text { [...] sendo retirada da DNDCD a }
$$

função de controle do câncer, desativada a CNCC e elaborado e aprovado novos organogramas e atribuições para o Instituto Nacional de Câncer, para o qual foi transferido o Programa de Oncologia (PRO-ONCO), agora sob a denominação de Coordenação de Programas de Controle de Câncer (PRO-ONCO) [...]. .

O êxito do PRO-ONCO, criado em 1986, consolidou-se quando o Programa passou a Coordenação de Programas de Controle de Câncer do INCA, em março deste ano.

Em 1996 surge a Coordenação Nacional de Controle do Tabagismo e Prevenção Primária do Câncer (CONTAPP), formada por um grupo de 27 profissionais responsáveis pela coordenação estadual do Programa, cujo objetivo era capacitar recursos humanos, desenvolver pesquisas, elaborar livros didáticos, folhetos, cartazes e adesivos sobre o assunto, prestar assessoria técnica nos projetos de lei relacionados ao tabagismo, em tramitação no Congresso Nacional, e participar de congressos de todas as áreas de conhecimento sobre o tema. A CONTAPP visava atender a necessidade de realização de um trabalho mais amplo sobre tabagismo, uma luta que o INCA havia incorporado participando de inúmeras campanhas e de eventos realizados, destacando-se o Congresso Brasileiro de Tabagismo.

Dentre as açôes desenvolvidas, cabe destacar a comemoração de datas específicas, como Dia Mundial do Tabaco (31 de maio) e o Dia Nacional de Combate 
ao Fumo (29 de agosto). A elaboração do Programa do Câncer Precoce abordando os fatores de risco comuns, a exemplo de exposição aos raios solares, hábitos alimentars inadequados, alcoolismo, fatores biológicos, poluição ocupacional e tabagismo. O empenho e o trabalho realizado pela CONTAPP resultou em que o INCA foi escolhido pela Organização Mundial de Saúde (OMS) para comandar todas as ações de controle do tabagismo nos países de língua portuguesa e espanhola na América Latina, Europa e África.

Açôes de destaque do INCA como a prevenção e a detecção precoce do câncer, passaram a ser desenvolvidas posteriormente pela Coordenação Nacional de Controle do Tabagismo, de Prevenção e Vigilância (CONPREV), que resultou da unificação de duas áreas de trabalho: o PRO-ONCO e a CONTAPP. A junção destas duas Unidades ocorreu durante o mandato de Jacob Kligerman como diretor do INCA, época em que foram criadas as Câmaras de Atividades Administrativas, divididas em quatro células: de Assistência, de Educação e Pesquisa, de Programas Nacionais e Estratégia.

O Programa Controle do Câncer de Colo Uterino Viva a Mulher, implantado em 1996, abrangia a detecção e o controle do câncer de colo de útero. O objetivo era prestar um serviço rápido, eficiente e capaz de atender as mulheres, informando-as e incentivando-as a se submeterem aos exames preconizados na prevenção e detecção da doença. Este programa consolidou-se em 1999, após a realização de campanhas, contando ainda com a assessoria do Cancer Care International (CCI), um setor da Fundação Ontário para tratamento e Pesquisa do Câncer, no Canadá.

Ressalta-se que enquanto o Programa de Controle de Câncer de Mama permaneceu em fase de implantação e desenvolvimento, o Programa de Controle do Câncer do Colo do Útero, em 2002, já tinha sido nacionalmente implantado, sendo considerado eficaz e atuante, tanto gerencial como operacionalmente, em todos os estados do Brasil.

O Projeto de Expansão da Assistência Oncológica no Brasil - EXPANDE, criado em 1998 e aprovado pelo Ministério da Saúde em 2000, objetivava implantar vinte Centros de Alta Complexidade em Oncologia (CACON) e, assim, aumentar a capacidade instalada de atendimento integral da rede de serviços do SUS. Os CACON são unidades hospitalares públicas ou filantrópicas que dispõem de recursos humanos e tecnológicos necessários à assistência ao paciente oncológico.

A CONPREV são atribuídos o desenvolvimento e o acompanhamento do Programa de Controle do Tabagismo e outros Fatores de Risco; do Programa de Controle do Câncer de Colo de Útero e Mama; do Programa de Epidemiologia e Vigilância do Câncer e seus Fatores de Risco; e do Programa EXPANDE.

A partir de 2000, por Decreto Presidencial no 3.496, o INCA passou a ter competência para desenvolver "açōes nacionais de controle do câncer", tornando-se referência nacional para a prestação de serviços oncológicos no âmbito do Sistema Único de Saúde SUS. ${ }^{10}$ Com base neste Decreto, foram criadas as cinco Unidades assistenciais descritas a seguir, independentes administrativamente e com equipes de saúde próprias, que permitiram a descentralização dos serviços prestados:

- Hospital do Câncer I (HC-I) - situado na Praça Cruz Vermelha, no 23, Centro, RJ, era a principal unidade hospitalar e também a de maior complexidade tecnológica. Localizada na sede do Instituto, atendia doentes portadores de todas as modalidades de câncer. - Hospital do Câncer II (HC-II) - ex-Hospital de Oncologia do INAMPS, localizado na rua do Equador, no 831, Santo Cristo, RJ, era o responsável pelo atendimento ambulatorial e de internações de adultos matriculados nos Serviços de Ginecologia e Oncologia Clínica.

- Hospital do Câncer III (HC-III) - ex-Hospital Luiza Gomes de Lemos, também conhecido como Hospital das Pioneiras Sociais, com sede na rua Visconde de Santa Isabel, no 274 , Vila Isabel, RJ, destinado ao diagnóstico e tratamento do câncer de mama.

- Hospital do Câncer IV (HC-IV) - Centro de Suporte Terapêutico Oncológico (CSTO), ex-Serviço Terapêutico Oncológico (STO), localizado na rua Visconde de Santa Isabel, no 274, Vila Isabel, RJ, em prédio anexo, em funcionamento desde 1987 com a finalidade de oferecer cuidados paliativos aos pacientes fora de possibilidades terapêuticas. Tinha como principais objetivos o atendimento domiciliar a estes doentes e a capacitação de profissionais para atuar nesta área.

- Centro Nacional de Transplante de Medula Óssea (CEMO) - destacava-se como o maior do Brasil no tratamento de doenças hematológicas, como a anemia aplástica severa e as leucemias. Realizava transplantes de medula óssea alogênicos e autogênicos, atendendo doentes do Rio de Janeiro e das demais regiōes do Brasil, no âmbito do SUS. O CEMO abrigava, também, o Registro Nacional de Doadores de Medula Óssea (REDOME) e o Banco de Células de Sangue de Cordão Umbilical e Placentário (BSCUP), além de centralizar as consultas aos bancos internacionais de doadores de medula óssea, ocupando parte do $7^{\circ}$ andar da sede do HC-I..$^{10}$

Deve-se ressaltar que os serviços descritos continuam sendo prestados pelas citadas Unidades, observados os avanços científicos relacionados ao diagnóstico e tratamento do câncer. 
A leitura do texto permite concluir que, no período enfocado, ocorreram importantes avanços técnicocientíficos e grandes realizaçôes na área de Oncologia, propiciando maior visibilidade às ações desenvolvidas pelo Instituto Nacional de Câncer, contribuindo para que a Instituição fosse reconhecida como referência para o tratamento de neoplasias, respeitada nacional e internacionalmente pela inequívoca competência de seus dirigentes, colaboradores e de todos aqueles que nela exercem atividades profissionais, tornando realidade o sonho de seus idealizadores.

\section{REFERÊNCIAS}

1. Instituto Nacional de Câncer; Ministério da Saúde, Secretaria Nacional de Saúde. Controle do câncer: uma proposta de integração ensino-serviço. 3a ed. Rio de Janeiro (Brasil): INCA; 1999.

2. Kroeff M. Resenha da luta contra o câncer no Brasil. Documentário do Serviço Nacional de Câncer. Rio de Janeiro (Brasil): Imprensa Brasileira; 1946.
3. Mello CG. O sistema de saúde em crise. 2a ed. São Paulo: CEBES: HUCITEC; 1981. (Coleção Saúde em Debate).

4. Instituto Nacional de Câncer; Ministério da Saúde, Secretaria Nacional de Assistência à Saúde. Ações de enfermagem para o controle do câncer. Rio de Janeiro (Brasil): PRO-ONCO; 1995.

5. Bodstein RCA, Fonseca CMO, Klein LE, Albuquerque MBM, Fernandes TMD. História da Saúde Pública: a política de controle do câncer no Brasil. Rio de Janeiro (Brasil): PEC: ENSP; 1987.

6. Piletti N. História do Brasil. 14a ed. São Paulo: Ática; 1997.

7. Mello CG. A medicina e a realidade brasileira. Rio de Janeiro: Achiamé; 1983.

8. Guimarães DS. Breve histórico do Instituto Nacional de Câncer desde sua criação até a presente data [mimeografado]. Rio de Janeiro: 2000.

9. Guimarães R, Tavares R, organizadores. Saúde e sociedade no Brasil: anos 80. Rio de Janeiro: Relume-Dumará; 1994.

10. Instituto Nacional de Câncer; Ministério da Saúde. Ações de enfermagem para o controle do câncer: uma proposta de integração ensino-serviço. 2a ed. Rio de Janeiro (Brasil): INCA; 2002 